\title{
New Inequalities for Preinvex Functions
}

\author{
Huriye Kadakal* and İmdat İşcan
}

\begin{abstract}
In this study, a new identity for functions defined on an open invex subset of set of real numbers is formed. After that we established Hermite-Hadamard-like inequalities for this type of functions. Then, by using the this identity and the Hölder and Power mean integral inequalities we present new type integral inequalities for functions whose powers of fourth derivatives in absolute value are preinvex functions.
\end{abstract}

Keywords: Invex set; preinvex function; Hölder Integral inequality.

AMS Subject Classification (2010): 26A51; 26D10; $26 D 15$.

\section{Preliminaries and fundamentals}

Definition 1.1. A function $\psi: I \subseteq \mathbb{R} \rightarrow \mathbb{R}$ is said to be convex if the inequality

$$
\psi(t \zeta+(1-t) \theta) \leq t \psi(\zeta)+(1-t) \psi(\theta)
$$

is valid for all $\zeta, \theta \in I$ and $t \in[0,1]$. If this inequality reverses, then $\psi$ is said to be concave on interval $I \neq \varnothing$.

Definition 1.2. $\psi: I \subseteq \mathbb{R} \rightarrow \mathbb{R}$ be a convex function on the interval $I$ of real numbers and $\zeta, \theta \in I$ with $\zeta<\theta$. The following celebrated double inequality

$$
\psi\left(\frac{\zeta+\theta}{2}\right) \leq \frac{1}{\theta-\zeta} \int_{\zeta}^{\theta} \psi(x) d x \leq \frac{\psi(\zeta)+\psi(\theta)}{2}
$$

is Hermite-Hadamard's inequality for convex functions [14]. Both inequalities hold in the reserved direction if $\psi$ is concave.

Hermite-Hadamard inequality [3] has been considered the most useful inequality in mathematical analysis. Readers can find more informations in $[2,5,8,14]$. Some of the classical inequalities for means can be derived from Hermite-Hadamard inequality for particular choices of $\psi$.

Let us recall the notion of preinvexity which is signicant generalizations of the notion of convexity, and some related results.

Definition 1.3 ([16]). Let $K$ be a non-empty subset in $\mathbb{R}^{n}$ and $\Lambda: K \times K \rightarrow \mathbb{R}$. Let $\zeta \in K$, then the set $K$ is said to be invex at $\zeta$ with respect to $\Lambda(\cdot, \cdot)$, if $\zeta+t \Lambda(\theta, \zeta) \in K, \quad \forall \zeta, \theta \in K \quad t \in[0,1] . K$ is said to be an invex set with respect to if $K$ is invex at each $\zeta \in K$. The invex set $K$ is also called $\Lambda$-connected set.

Definition 1.4 ([16]). A function $\psi: K \rightarrow \mathbb{R}$ on an invex set $K \subseteq \mathbb{R}$ is said to be preinvex with respect to $\eta$, if

$$
\psi(\zeta+t \Lambda(\theta, \zeta)) \leq(1-t) \psi(\zeta)+t \psi(\theta), \quad \forall \zeta, \theta \in K, \quad t \in[0,1]
$$

The function $\psi$ is said to be preconcave if and only if $-\psi$ is preinvex.

Received : 30-10-2018, Accepted : 06-03-2019 
It is to be noted that every convex function is preinvex with respect to the map $\Lambda(\theta, \zeta)=\theta-\zeta$ but the converse is not true see for instance.

In [10], the authors introduced Condition $C$ defined as follows:

Definition 1.5 ([10]). Let $S \subseteq \mathbb{R}$ be an open invex subset with respect to the map $\Lambda: S \times S \rightarrow \mathbb{R}$. We say that the function satisfies the Condition $C$ if, for any $\zeta, \theta \in S$ and any $t \in[0,1]$,

$$
\begin{gathered}
\Lambda(\theta, \theta+t \Lambda(\zeta, \theta))=-t \Lambda(\zeta, \theta) \\
\Lambda(\zeta, \theta+t \Lambda(\zeta, \theta))=(1-t) \Lambda(\zeta, \theta) .
\end{gathered}
$$

Note that, using the Condition $C$, we have

$$
\Lambda\left(\theta+t_{2} \Lambda(\zeta, \theta) \theta, \theta+t_{1} \Lambda(\zeta, \theta)\right)=\left(t_{2}-t_{1}\right) \Lambda(\zeta, \theta)
$$

for any $\zeta, \theta \in S$ and any $t_{1}, t_{2} \in[0,1]$.

In recent years, many mathematicians have been studying about preinvexity and types of preinvexity. A lot of efforts have been made by many mathematicians to generalize the classical convexity [4, 12, 13, 15-17]. Ben-Israel and Mond gave the concept of preinvex functions which is a special case of invexity [4].

In [11], Noor has obtained the following Hermite-Hadamard type inequalities for the preinvex functions.

Theorem 1.1 ([11]). Let $\psi:[\zeta, \zeta+\Lambda(\theta, \zeta)] \rightarrow(0, \infty)$ be a preinvex function on the interval of the real numbers $K^{\circ}$ (the interior of $K$ ) and $\zeta, \theta \in K^{\circ}$ with $\Lambda(\theta, \zeta)>0$. Then the following inequalities holds

$$
\psi\left(\frac{2 \zeta+\eta(\theta, \zeta)}{2}\right) \leq \frac{1}{\Lambda(\theta, \zeta)} \int_{\zeta}^{\zeta+\Lambda(\theta, \zeta)} \psi(x) d x \leq \frac{\psi(\zeta)+\psi(\theta)}{2}
$$

For several recent results on inequalities for preinvex functions which are connected to (1.3), we refer the interested reader to $[1,6,7,9]$ and the references therein.

Let $0<\zeta<\theta$, throughout this paper we will use

$$
\begin{aligned}
A & =A(\zeta, \theta)=\frac{\zeta+\theta}{2} \\
L_{p}(\zeta, \theta) & =\left(\frac{\theta^{p+1}-\zeta^{p+1}}{(p+1)(\theta-\zeta)}\right)^{\frac{1}{p}}, \zeta \neq \theta, p \in \mathbb{R}, p \neq-1,0
\end{aligned}
$$

for the arithmetic and generalized logarithmic mean, respectively. Moreover, for shortness, the following notations will be used:

$$
\begin{array}{ll}
\alpha=\alpha(\zeta, \theta, \Lambda)=\zeta+\frac{\Lambda(\theta, \zeta)}{2}, & \alpha_{t}=\alpha_{t}(\zeta, \theta, \Lambda)=\zeta+t \frac{\Lambda(\theta, \zeta)}{2} \\
\beta=\beta(\zeta, \theta, \Lambda)=\zeta+\frac{\Lambda(\theta, \zeta)}{3}, & \beta_{t}=\beta_{t}(\zeta, \theta, \Lambda)=\zeta+t \frac{\Lambda(\theta, \zeta)}{3} \\
\gamma=\beta(\zeta, \theta, \Lambda)=\zeta+\frac{\Lambda(\theta, \zeta)}{4}, & \gamma_{t}=\gamma_{t}(\zeta, \theta, \Lambda)=\zeta+t \frac{\Lambda(\theta, \zeta)}{4}
\end{array}
$$

and

$$
\begin{aligned}
& I_{\psi}(\zeta, \theta, \Lambda):=\frac{\Lambda^{3}(\theta, \zeta)}{6}\left(\zeta+\frac{\Lambda(\theta, \zeta)}{4}\right) \psi^{\prime \prime \prime}(\zeta+\Lambda(\theta, \zeta))-\frac{\Lambda^{2}(\theta, \zeta)}{2}\left(\zeta+\frac{\Lambda(\theta, \zeta)}{3}\right) \psi^{\prime \prime}(\zeta+\Lambda(\theta, \zeta)) \\
& +\Lambda(\theta, \zeta)\left(\zeta+\frac{\Lambda(\theta, \zeta)}{2}\right) \psi^{\prime}(\zeta+\Lambda(\theta, \zeta))-(\zeta+\Lambda(\theta, \zeta)) \psi(\zeta+\Lambda(\theta, \zeta))+\Lambda(\theta, \zeta) \int_{0}^{1} \psi(\zeta+t \Lambda(\theta, \zeta)) d t
\end{aligned}
$$

In this paper, using a general integral identity for a three times differentiable functions, we establish some new type integral inequalities for mappings whose third derivative in absolute value at certain powers are preinvex. 


\section{Main result for our lemma}

We will use the following Lemma for obtain our main results about the preinvexity.

Lemma 2.1. Let $K \subseteq \mathbb{R}$ be an open invex subset with respect to mapping $\Lambda(\cdot, \cdot): K \times K \rightarrow \mathbb{R}$ and $\zeta, \theta \in K$ with $\Lambda(\theta, \zeta)>0$. Suppose that the function $\psi: K \rightarrow \mathbb{R}$ is a fourth times differentiable function on $K$ such that $\psi^{(i v)} \in L[\zeta, \zeta+\Lambda(\theta, \zeta)]$. Then the following identity hold:

$$
\begin{aligned}
& \Lambda^{4}(\theta, \zeta) \int_{0}^{1} \frac{t^{3}}{6}\left(\zeta+t \frac{\Lambda(\theta, \zeta)}{4}\right) \psi^{(i v)}(\zeta+t \Lambda(\theta, \zeta)) d t=\frac{\Lambda^{3}(\theta, \zeta)}{6} \gamma \psi^{\prime \prime \prime}(\zeta+\Lambda(\theta, \zeta))-\frac{\Lambda^{2}(\theta, \zeta)}{2} \beta \psi^{\prime \prime}(\zeta+\Lambda(\theta, \zeta)) \\
& +\Lambda(\theta, \zeta) \alpha \psi^{\prime}(\zeta+\Lambda(\theta, \zeta))-\psi(\zeta+\Lambda(\theta, \zeta))(\zeta+\Lambda(\theta, \zeta))+\psi(\zeta) \zeta+\int_{\zeta}^{\zeta+\Lambda(\theta, \zeta)} \psi(u) d u
\end{aligned}
$$

Proof. Integrating fourth times by parts and then changing the variable, we obtain

$$
\begin{aligned}
& \Lambda^{4}(\theta, \zeta) \int_{0}^{1} \frac{t^{3}}{6}\left(\zeta+t \frac{\Lambda(\theta, \zeta)}{4}\right) \psi^{(i v)}(\zeta+t \Lambda(\theta, \zeta)) d t \\
= & \left.\Lambda^{3}(\theta, \zeta) \frac{t^{3}}{6} \gamma_{t} \psi^{\prime \prime \prime}(\zeta+t \Lambda(\theta, \zeta))\right|_{0} ^{1}-\left.\Lambda^{2}(\theta, \zeta) \frac{t^{2}}{2} \beta_{t} \psi^{\prime \prime}(\zeta+t \Lambda(\theta, \zeta))\right|_{0} ^{1} \\
& +\left.\Lambda(\theta, \zeta) t \alpha_{t} \psi^{\prime}(\zeta+t \Lambda(\theta, \zeta))\right|_{0} ^{1}-\left.(\zeta+t \Lambda(\theta, \zeta)) \psi(\zeta+t \Lambda(\theta, \zeta))\right|_{0} ^{1} \\
& +\Lambda(\theta, \zeta) \int_{0}^{1} \psi(\zeta+t \Lambda(\theta, \zeta)) d t \\
= & \frac{\Lambda^{3}(\theta, \zeta)}{6} \gamma \psi^{\prime \prime \prime}(\zeta+\Lambda(\theta, \zeta))-\frac{\Lambda^{2}(\theta, \zeta)}{2} \beta \psi^{\prime \prime}(\zeta+\Lambda(\theta, \zeta)) \\
& +\Lambda(\theta, \zeta) \alpha \psi^{\prime}(\zeta+\Lambda(\theta, \zeta))-(\zeta+\Lambda(\theta, \zeta)) \psi(\zeta+\Lambda(\theta, \zeta)) \\
& +\zeta \psi(\zeta)+\Lambda(\theta, \zeta) \int_{0}^{1} \psi(\zeta+t \Lambda(\theta, \zeta)) d t \\
= & \frac{\Lambda^{3}(\theta, \zeta)}{6} \gamma \psi^{\prime \prime \prime}(\zeta+\Lambda(\theta, \zeta))-\frac{\Lambda^{2}(\theta, \zeta)}{2} \beta \psi^{\prime \prime}(\zeta+\Lambda(\theta, \zeta)) \\
& +\Lambda(\theta, \zeta) \alpha \psi^{\prime}(\zeta+\Lambda(\theta, \zeta))-(\zeta+\Lambda(\theta, \zeta)) \psi(\zeta+\Lambda(\theta, \zeta)) \\
& +\zeta \psi(\zeta)+\int_{\zeta}^{\zeta+\Lambda(\theta, \zeta)} \psi(x) d x
\end{aligned}
$$

Theorem 2.1. Let $K \subseteq \mathbb{R}$ be an open invex subset with respect to mapping $\Lambda(\cdot, \cdot): K \times K \rightarrow \mathbb{R}$ and $\zeta, \theta \in K$ with $\Lambda(\theta, \zeta)>$ 0 . Suppose that the function $\psi: K \rightarrow \mathbb{R}$ is a fourth times differentiable function on $K$ such that $\psi^{(2 v)} \in L[\zeta, \zeta+\Lambda(\theta, \zeta)]$. If $\left|\psi^{(\imath v)}\right|^{q}$ is preinvex on $K$ for $q>1$, then the following inequality holds:

$$
\left|I_{\psi}(\zeta, \theta, \Lambda)\right| \leq \frac{2^{1-\frac{2}{p}}}{3} \frac{\Lambda^{2+\frac{2}{p}}(\theta, \zeta)}{(3 p+1)^{\frac{1}{p}}}\left[\left|\psi^{(i v)}(\theta)\right|^{q} C_{1, \Lambda}(\zeta, \theta)+\left|\psi^{(i v)}(\zeta)\right|^{q} C_{2, \Lambda}(\zeta, \theta)\right]^{\frac{1}{q}} .
$$

where

$$
C_{1, \Lambda}(\zeta, \theta):=\left\{\begin{array}{cc}
\Lambda(\theta, \zeta)\left[L_{q+1}^{q+1}(\gamma, \zeta)-\zeta L_{q}^{q}(\gamma, \zeta)\right], & \zeta>0, \gamma>0, \\
4(\zeta+\gamma) L_{q+1}^{q+1}(\gamma,-\zeta)-\frac{8 \zeta}{q+1} A\left(\gamma^{q+1},(-\zeta)^{q+1}\right), \zeta<0, \gamma>0 \\
-\Lambda(\theta, \zeta)\left[L_{q+1}^{q+1}(-\zeta,-\gamma)+\zeta L_{q}^{q}(-\zeta,-\gamma)\right], & \zeta<0, \gamma<0
\end{array}\right.
$$

and

$$
C_{2, \Lambda}(\zeta, \theta):=\left\{\begin{array}{cc}
-\Lambda(\theta, \zeta)\left[L_{q+1}^{q+1}(\gamma, \zeta)-\gamma L_{q}^{q}(\gamma, \zeta)\right], & \zeta>0, \gamma>0, \\
-4(\zeta+\gamma) L_{q+1}^{q+1}(\gamma,-\zeta)+\frac{8 \gamma}{q+1} A\left(\gamma^{q+1},(-\zeta)^{q+1}\right), \zeta<0, \gamma>0, & \\
\Lambda(\theta, \zeta)\left[L_{q+1}^{q+1}(-\zeta,-\gamma)+\gamma L_{q}^{q}(-\zeta,-\gamma)\right], & \zeta<0, \gamma<0 .
\end{array}\right.
$$


Proof. If $\left|\psi^{(i v)}\right|^{q}$ for $q>1$ is preinvex on $[\zeta, \zeta+\Lambda(\theta, \zeta)]$, using Lemma 2.1, the Hölder integral inequality and $\left|\psi^{(i v)}(\zeta+t \Lambda(\theta, \zeta))\right|^{q} \leq t\left|\psi^{(i v)}(\theta)\right|^{q}+(1-t)\left|\psi^{(i v)}(\zeta)\right|^{q}$, we get

$$
\begin{aligned}
& \left|I_{\psi}(\zeta, \theta, \Lambda)\right| \\
\leq & \frac{1}{6} \Lambda^{4}(\theta, \zeta) \int_{0}^{1} t^{3}\left|\gamma_{t}\right|\left|\psi^{(i v)}(\zeta+t \Lambda(\theta, \zeta))\right| d t \\
\leq & \frac{1}{6} \Lambda^{4}(\theta, \zeta)\left(\int_{0}^{1} t^{3 p} d t\right)^{\frac{1}{p}}\left(\int_{0}^{1}\left|\gamma_{t}\right|^{q}\left|\psi^{(i v)}(\zeta+t \Lambda(\theta, \zeta))\right|^{q} d t\right)^{\frac{1}{q}} \\
\leq & \frac{1}{6} \Lambda^{4}(\theta, \zeta)\left(\int_{0}^{1} t^{3 p} d t\right)^{\frac{1}{p}}\left(\int_{0}^{1}\left|\gamma_{t}\right|^{q}\left[t\left|\psi^{(i v)}(\theta)\right|^{q}+(1-t)\left|\psi^{(i v)}(\zeta)\right|^{q}\right] d t\right)^{\frac{1}{q}} \\
\leq & \frac{1}{6} \frac{\Lambda^{4}(\theta, \zeta)}{(3 p+1)^{\frac{1}{p}}}\left(\left|\psi^{(i v)}(\theta)\right|^{q} \int_{0}^{1} t\left|\gamma_{t}\right|^{q} d t+\left|\psi^{(i v)}(\zeta)\right|^{q} \int_{0}^{1}(1-t)\left|\gamma_{t}\right|^{q} d t\right)^{\frac{1}{q}} \\
= & \frac{1}{6} \frac{\Lambda^{4}(\theta, \zeta)}{(3 p+1)^{\frac{1}{p}}}\left(\frac{4\left|\psi^{(i v)}(\theta)\right|^{q}}{\Lambda^{2}(\theta, \zeta)} \int_{\zeta}^{\gamma} 4(u-\zeta)|u|^{q} d u+\frac{4\left|\psi^{(i v)}(\zeta)\right|^{q}}{\Lambda^{2}(\theta, \zeta)} \int_{\zeta}^{\gamma}(\Lambda(\theta, \zeta)-4(u-\zeta))|u|^{q} d u\right)^{\frac{1}{q}} \\
= & \frac{2^{1-\frac{2}{p}}}{3} \frac{\Lambda^{2+\frac{2}{p}}(\theta, \zeta)}{(3 p+1)^{\frac{1}{p}}}\left(\left|\psi^{(i v)}(\theta)\right|^{q} \int_{\zeta}^{\gamma} 4(u-\zeta)|u|^{q} d u+\left|\psi^{(i v)}(\zeta)\right|^{q} \int_{\zeta}^{\gamma}(\Lambda(\theta, \zeta)-4(u-\zeta))|u|^{q} d u\right)^{\frac{1}{q}} \\
= & \frac{2^{1-\frac{2}{p}}}{3} \frac{\Lambda^{2+\frac{2}{p}}(\theta, \zeta)}{(3 p+1)^{\frac{1}{p}}}\left[\left|\psi^{(i v)}(\theta)\right|^{q} C_{1, \Lambda}(\zeta, \theta)+\left|\psi^{(i v)}(\zeta)\right|^{q} C_{2, \Lambda}(\zeta, \theta)\right]^{\frac{1}{q}} \cdot
\end{aligned}
$$

Corollary 2.1. Under the conditions of Theorem 2.1, If we choose $\eta(\theta, \zeta)=\theta-\zeta$ then when $\left|\psi^{(i v)}\right|^{q}$ is convex on $K$ for $q>1$ we obtain

$$
\begin{aligned}
& \left|\frac{(\theta-\zeta)^{2}}{6}\left(\frac{3 \zeta+\theta}{4}\right) \psi^{\prime \prime \prime}(\theta)-\frac{(\theta-\zeta)}{2}\left(\frac{2 \zeta+\theta}{3}\right) \psi^{\prime \prime}(\theta)+\frac{\zeta+\theta}{2} f^{\prime}(\theta)-\frac{\psi(\theta) \theta-\psi(\zeta) \zeta}{\theta-\zeta}+\frac{1}{\theta-\zeta} \int_{\zeta}^{\theta} \psi(u) d u\right| \\
\leq & \frac{2^{1-\frac{2}{p}}}{3} \frac{(\theta-\zeta)^{1+\frac{2}{p}}}{(3 p+1)^{\frac{1}{p}}}\left[\left|\psi^{(\imath v)}(\theta)\right|^{q} C_{1, \eta}(\zeta, \theta)+\left|\psi^{(\imath v)}(\zeta)\right|^{q} C_{2, \eta}(\zeta, \theta)\right]^{\frac{1}{q}},
\end{aligned}
$$

where

$$
\begin{gathered}
C_{1}(\zeta, \theta)=\left\{\begin{array}{cc}
(\theta-\zeta)\left[L_{q+1}^{q+1}\left(\frac{3 \zeta+\theta}{4}, \zeta\right)-\zeta L_{q}^{q}\left(\frac{3 \zeta+\theta}{4}, \zeta\right)\right], & \zeta>0, \frac{3 \zeta+\theta}{4}>0, \\
(7 \zeta+\theta) L_{q+1}^{q+1}\left(\frac{3 \zeta+\theta}{4},-\zeta\right)-\frac{8 \zeta}{q+1} A\left(\left(\frac{3 \zeta+\theta}{4}\right)^{q+1},(-\zeta)^{q+1}\right), \zeta<0, \frac{3 \zeta+\theta}{4}>0, & \\
-(\theta-\zeta)\left[L_{q+1}^{q+1}\left(-\zeta,-\frac{3 \zeta+\theta}{4}\right)+\zeta L_{q}^{q}(-\zeta,-\gamma)\right], & \zeta<0, \frac{3 \zeta+\theta}{4}<0 .
\end{array}\right. \\
C_{2}(\zeta, \theta)=\left\{\begin{array}{cc}
-(\theta-\zeta)\left[L_{q+1}^{q+1}\left(\frac{3 \zeta+\theta}{4}, \zeta\right)-\gamma L_{q}^{q}\left(\frac{3 \zeta+\theta}{4}, \zeta\right)\right], & \zeta>0, \frac{3 \zeta+\theta}{4}>0, \\
-(7 \zeta+\theta) L_{q+1}^{q+1}\left(\frac{3 \zeta+\theta}{4},-\zeta\right)+\frac{8 \gamma}{q+1} A\left(\left(\frac{3 \zeta+\theta}{4}\right)^{q+1},(-\zeta)^{q+1}\right), \zeta<0, \frac{3 \zeta+\theta}{4}>0, & \zeta<0, \frac{3 \zeta+\theta}{4}<0 .
\end{array}\right.
\end{gathered}
$$

Remark 2.1. If the mapping $\Lambda$ satisfies condition $C$ then by use of the preinvexity of $\left|\psi^{(i v)}\right|^{q}$ we obtain following inequality for every $t \in[0,1]$ :

$$
\begin{aligned}
\left|\psi^{(i v)}(\zeta+t \Lambda(\theta, \zeta))\right|^{q} & =\left|\psi^{(i v)}(\zeta+\Lambda(\theta, \zeta)+(1-t) \Lambda(\zeta, \zeta+\Lambda(\theta, \zeta)))\right|^{q} \\
& \leq t\left|\psi^{(i v)}(\zeta+\Lambda(\theta, \zeta))\right|^{q}+(1-t)\left|\psi^{(i v)}(\zeta)\right|^{q} .
\end{aligned}
$$

If we use (2.2) in the proof of Theorem 2.1, then (2.1) becomes the following inequality:

$$
I_{\psi}(\zeta, \theta, \Lambda) \leq \frac{2^{1-\frac{2}{p}}}{3} \frac{\Lambda^{2+\frac{2}{p}}(\theta, \zeta)}{(3 p+1)^{\frac{1}{p}}} \times\left[\left|\psi^{(i v)}(\zeta+\Lambda(\theta, \zeta))\right|^{q} C_{1, \Lambda}(\zeta, \theta)+\left|\psi^{(i v)}(\zeta)\right|^{q} C_{2, \Lambda}(\zeta, \theta)\right]^{\frac{1}{q}} .
$$


We note that by use of the preinvexity of $\left|\psi^{(i v)}\right|^{q}$ we get $\left|\psi^{(i v)}(\zeta+\Lambda(\theta, \zeta))\right|^{q} \leq\left|\psi^{(i v)}(\theta)\right|^{q}$. Therefore, the inequality (2.3) is better than (2.1).

Theorem 2.2. Let $K \subseteq \mathbb{R}$ be an open invex subset with respect to mapping $\Lambda(\cdot, \cdot): K \times K \rightarrow \mathbb{R}$ and $\zeta, \theta \in K$ with $\Lambda(\theta, \zeta)>0$. Suppose that the function $\psi: K \rightarrow \mathbb{R}$ is a fourth times differentiable function on $K$ such that $\psi^{(i v)} \in L[\zeta, \zeta+\Lambda(\theta, \zeta)]$. If $\left|\psi^{(i v)}\right|^{q}$ is preinvex on $K$ for $q>1$, then the following inequality holds:

$$
\left|I_{\psi}(\zeta, \theta, \eta)\right| \leq \frac{2^{\frac{2}{p}-1}}{3} \Lambda^{3+\frac{1}{q}}(\theta, \zeta) C_{3, \Lambda}^{\frac{1}{p}}(\zeta, \theta)\left[\frac{(3 q+1)\left|\psi^{(i v)}(\theta)\right|^{q}+\left|\psi^{(i v)}(\zeta)\right|^{q}}{(3 q+1)(3 q+2)}\right]^{\frac{1}{q}}
$$

where

$$
C_{3, \Lambda}(\zeta, \theta)=\left\{\begin{array}{cc}
\frac{\Lambda(\theta, \zeta)}{2} L_{p}^{p}(\gamma, \zeta), & \zeta>0, \gamma>0 \\
\frac{2}{p+1} A\left(\gamma^{p+1},(-\zeta)^{p+1}\right), \zeta<0, & \gamma>0 \\
\frac{\Lambda(\theta, \zeta)}{4} L_{p}^{p}(-\zeta,-\gamma), & \zeta<0, \quad \gamma<0
\end{array}\right.
$$

Proof. If $\left|\psi^{(i v)}\right|^{q}$ for $q>1$ is preinvex on $[\zeta, \zeta+\Lambda(\theta, \zeta)]$, using Lemma 2.1, the Hölder integral inequality and $\left|\psi^{(i v)}(\zeta+t \Lambda(\theta, \zeta))\right|^{q} \leq t\left|\psi^{(i v)}(\theta)\right|^{q}+(1-t)\left|\psi^{(i v)}(\zeta)\right|^{q}$, we obtain the following inequality:

$$
\begin{aligned}
& \left|I_{f}(\zeta, \theta, \Lambda)\right| \\
\leq & \frac{1}{6} \Lambda^{4}(\theta, \zeta) \int_{0}^{1} t^{3}\left|\gamma_{t}\right|\left|\psi^{(i v)}(\zeta+t \Lambda(\theta, \zeta))\right| d t \\
\leq & \frac{1}{6} \Lambda^{4}(\theta, \zeta)\left(\int_{0}^{1}\left|\gamma_{t}\right|^{p} d t\right)^{\frac{1}{p}}\left(\int_{0}^{1} t^{3 q}\left|\psi^{(i v)}(\zeta+t \Lambda(\theta, \zeta))\right|^{q} d t\right)^{\frac{1}{q}} \\
\leq & \frac{1}{6} \Lambda^{4}(\theta, \zeta)\left(\int_{0}^{1}\left|\gamma_{t}\right|^{p} d t\right)^{\frac{1}{p}}\left(\int_{0}^{1} t^{3 q}\left[t\left|\psi^{(i v)}(\theta)\right|^{q}+(1-t)\left|\psi^{(i v)}(\zeta)\right|^{q}\right] d t\right)^{\frac{1}{q}} \\
= & \frac{1}{6} \Lambda^{4}(\theta, \zeta)\left(\int_{0}^{1}\left|\gamma_{t}\right|^{p} d t\right)^{\frac{1}{p}}\left(\left|\psi^{(i v)}(\theta)\right|^{q} \int_{0}^{1} t^{3 q+1} d t+\left|\psi^{(i v)}(\zeta)\right|^{q} \int_{0}^{1}\left(t^{3 q}-t^{3 q+1}\right) d t\right)^{\frac{1}{q}} \\
= & \frac{1}{3} 2^{\frac{2}{p}-1} \Lambda^{3+\frac{1}{q}}(\theta, \zeta)\left(\int_{a}^{\gamma}|x|^{p} d x\right)^{\frac{1}{p}}\left[\frac{\left|\psi^{(i v)}(\theta)\right|^{q}}{3 q+2}+\left|\psi^{(i v)}(\zeta)\right|^{q}\left(\frac{1}{3 q+1}-\frac{1}{3 q+2}\right)\right]^{\frac{1}{q}} \\
= & \frac{1}{3} 2^{\frac{2}{p}-1} \Lambda^{3+\frac{1}{q}}(\theta, \zeta) C_{3, \Lambda}^{\frac{1}{p}}(\zeta, \theta)\left[\frac{(3 q+1)\left|\psi^{(i v)}(\theta)\right|^{q}+\left|\psi^{(i v)}(\zeta)\right|^{q}}{(3 q+1)(3 q+2)}\right]^{\frac{1}{q}}
\end{aligned}
$$

Corollary 2.2. Under the conditions of Theorem 2.2, If we choose $\Lambda(\theta, \zeta)=\theta-\zeta$ then when $\left|\psi^{(i v)}\right|^{q}$ is convex on $K$ for $q>1$ we have

$$
\begin{aligned}
& \left|\frac{(\theta-\zeta)^{2}}{6}\left(\frac{3 \zeta+\theta}{4}\right) \psi^{\prime \prime \prime}(\theta)-\frac{(\theta-\zeta)}{2}\left(\frac{2 \zeta+\theta}{3}\right) \psi^{\prime \prime}(\theta)+\frac{\zeta+\theta}{2} \psi^{\prime}(\theta)-\frac{\psi(\theta) \theta-\psi(\zeta) \zeta}{\theta-\zeta}+\frac{1}{\theta-\zeta} \int_{\zeta}^{\theta} \psi(u) d u\right| \\
\leq & \frac{1}{3} 2^{\frac{2}{p}-1}(\theta-\zeta)^{2+\frac{1}{q}} C_{3, \Lambda}^{\frac{1}{p}}(\zeta, \theta)\left[\frac{(3 q+1)\left|\psi^{(i v)}(\theta)\right|^{q}+\left|\psi^{(i v)}(\zeta)\right|^{q}}{(3 q+1)(3 q+2)}\right]^{\frac{1}{q}},
\end{aligned}
$$

where

$$
C_{3}(\zeta, \theta)=\left\{\begin{array}{cl}
\frac{\theta-\zeta}{4} L_{p}^{p}\left(\frac{3 \zeta+\theta}{4}, \zeta\right), & \zeta>0, \frac{3 \zeta+\theta}{4}>0 \\
\frac{2}{p+1} A\left(\left(\frac{3 \zeta+\theta}{4}\right)^{p+1},(-\zeta)^{p+1}\right), \zeta<0, \frac{3 \zeta+\theta}{4}>0 \\
\frac{\theta-\zeta}{4} L_{p}^{p}\left(-\zeta,-\frac{3 \zeta+\theta}{4}\right), & \zeta<0, \frac{3 \zeta+\theta}{4}<0
\end{array}\right.
$$


Remark 2.2. If the mapping $\Lambda$ satisfies condition $C$ then using (2.2) in the proof of Theorem 2.2, then (2.4) becomes the following inequality:

$$
\left|I_{\psi}(\zeta, \theta, \Lambda)\right| \leq \frac{1}{3} 2^{\frac{2}{p}-1} \Lambda^{3+\frac{1}{q}}(\theta, \zeta) C_{3, \Lambda}^{\frac{1}{p}}(\zeta, \theta)\left[\frac{(3 q+1)\left|\psi^{(i v)}(\zeta+t \Lambda(\theta, \zeta))\right|^{q}+\left|\psi^{(i v)}(\zeta)\right|^{q}}{(3 q+1)(3 q+2)}\right]^{\frac{1}{q}} .
$$

We note that by use of the preinvexity of $\left|\psi^{(i v)}\right|^{q}$ we get $\left|\psi^{(i v)}(\zeta+\Lambda(\theta, \zeta))\right|^{q} \leq\left|\psi^{(i v)}(\theta)\right|^{q}$. Therefore, (2.5) is better than (2.4).

Theorem 2.3. Let $K \subseteq \mathbb{R}$ be an open invex subset with respect to mapping $\Lambda(\cdot, \cdot): K \times K \rightarrow \mathbb{R}$ and $\zeta, \theta \in K$ with $\Lambda(\theta, \zeta)>$ 0 . Suppose that the function $\psi: K \rightarrow \mathbb{R}$ is a fourth times differentiable function on $K$ such that $\psi^{(i v)} \in L[\zeta, \zeta+\Lambda(\theta, \zeta)]$. If $\left|\psi^{(i v)}\right|^{q}$ is preinvex on $K$ for $q \geq 1$, then the following inequality holds:

$$
\left|I_{\psi}(\zeta, \theta, \Lambda)\right| \leq \frac{2^{7}}{3} \Lambda^{-\frac{1}{q}}(\theta, \zeta) D_{1, \Lambda}^{1-\frac{1}{q}}(\zeta, \theta)\left[\left|\psi^{(i v)}(\theta)\right|^{q} D_{2, \Lambda}(\zeta, \theta)+\left|\psi^{(i v)}(\zeta)\right|^{q} D_{3, \Lambda}(\zeta, \theta)\right]^{\frac{1}{q}}
$$

where

$$
\begin{aligned}
& D_{1, \Lambda}(\zeta, \theta):= \begin{cases}\frac{\Lambda^{4}(\theta, \zeta)}{4^{4}} \frac{\Lambda(\theta, \zeta)+5 \zeta}{20}, & \zeta>0, \gamma>0 \\
\frac{\Lambda^{4}(\theta, \zeta)}{4^{4}} \frac{\Lambda(\theta, \zeta)+5 \zeta}{20}-\frac{\zeta^{5}}{10}, \zeta<0, \gamma>0 & \\
-\frac{\Lambda^{4}(\theta, \zeta)}{4^{4}} \frac{\Lambda(\theta, \zeta)+5 \zeta}{20}, & \zeta<0, \gamma<0\end{cases} \\
& D_{2, \Lambda}(\zeta, \theta):= \begin{cases}\frac{\Lambda^{5}(\theta, \zeta)}{4^{5}} \frac{\Lambda(\theta, \zeta)+24 \zeta}{120}, & \zeta>0, \gamma>0 \\
\frac{\Lambda^{5}(\theta, \zeta)}{4^{4}} \frac{5 \Lambda(\theta)+24 \zeta}{120}+\frac{\zeta^{6}}{15}, \zeta<0, \gamma>0 & \zeta \Lambda^{5}(\theta, \zeta) \\
-\frac{\Lambda(\theta, \zeta)+24 \zeta}{120}, & \zeta<0, \gamma<0\end{cases} \\
& D_{3, \Lambda}(\zeta, \theta):= \begin{cases}\frac{\Lambda^{5}(\theta, \zeta)}{4^{4}} \frac{\Lambda(\theta, \zeta)+6 \zeta}{120}, & \zeta>0, \gamma>0 \\
\frac{\Lambda^{5}(\theta, \zeta)}{\Lambda 4^{4}} \frac{\Lambda(\theta, \zeta)+5 \zeta}{60}-\Lambda(\theta, \zeta) \frac{\zeta^{5}}{10}-4 \frac{\zeta^{6}}{15}, \zeta<0, \gamma>0 \\
-\frac{\Lambda^{5}(\theta, \zeta)}{4^{4}} \frac{\Lambda(\theta, \zeta)+6 \zeta}{120}, & \zeta<0, \gamma<0 .\end{cases}
\end{aligned}
$$

Proof. Using Lemma 2.1 and Power-mean integral inequality, we get

$$
\begin{aligned}
& \left|I_{\psi}(\zeta, \theta, \Lambda)\right| \\
& \leq \frac{1}{6} \Lambda^{4}(\theta, \zeta) \int_{0}^{1} t^{3}\left|\gamma_{t}\right|\left|\psi^{(i v)}(\zeta+t \Lambda(\theta, \zeta))\right| d t \\
& \leq \frac{1}{6} \Lambda^{4}(\theta, \zeta)\left(\int_{0}^{1} t^{3}\left|\gamma_{t}\right| d t\right)^{1-\frac{1}{q}}\left(\int_{0}^{1} t^{3}\left|\gamma_{t}\right|\left|\psi^{(i v)}(\zeta+\Lambda(\theta, \zeta))\right|^{q} d t\right)^{\frac{1}{q}} \\
& \leq \frac{1}{6} \Lambda^{4}(\theta, \zeta)\left(\int_{0}^{1} t^{3}\left|\gamma_{t}\right| d t\right)^{1-\frac{1}{q}}\left(\int_{0}^{1} t^{3}\left|\gamma_{t}\right|\left[t\left|\psi^{(i v)}(\theta)\right|^{q}+(1-t)\left|\psi^{(i v)}(\zeta)\right|^{q}\right] d t\right)^{\frac{1}{q}} \\
& =\frac{1}{6} \Lambda^{4}(\theta, \zeta)\left(\int_{0}^{1} t^{3}\left|\gamma_{t}\right| d t\right)^{1-\frac{1}{q}}\left(\left|\psi^{(i v)}(\theta)\right|^{q} \int_{0}^{1} t^{4}\left|\gamma_{t}\right| d t+\left|\psi^{(i v)}(\zeta)\right|^{q} \int_{0}^{1} t^{3}(1-t)\left|\gamma_{t}\right| d t\right)^{\frac{1}{q}} \\
& =\frac{1}{6} \Lambda^{4}(\theta, \zeta)\left(\frac{4^{4}}{\Lambda^{4}(\theta, \zeta)}\right)^{1-\frac{1}{q}}\left(\frac{4^{4}}{\Lambda^{5}(\theta, \zeta)}\right)^{\frac{1}{q}}\left(\int_{\zeta}^{\gamma}(u-\zeta)^{3}|u| d u\right)^{1-\frac{1}{q}} \\
& \times\left(\left|\psi^{(i v)}(\theta)\right|^{q} \int_{\zeta}^{\gamma} 4(u-\zeta)^{4}|u| d u+\left|\psi^{(i v)}(\zeta)\right|^{q} \int_{\zeta}^{\gamma}(u-\zeta)^{3}[\Lambda(\theta, \zeta)-4(u-\zeta)]|u| d x\right)^{\frac{1}{q}} \\
& =\frac{2^{7}}{3} \eta^{-\frac{1}{q}}(\theta, \zeta)\left(\int_{\zeta}^{\gamma}(u-\zeta)^{3}|u| d u\right)^{1-\frac{1}{q}} \\
& \times\left(\left|\psi^{(i v)}(\theta)\right|^{q} \int_{\zeta}^{\gamma} 4(u-\zeta)^{4}|u| d u+\left|\psi^{(i v)}(\zeta)\right|^{q} \int_{\zeta}^{\gamma}(u-\zeta)^{3}[\Lambda(\theta, \zeta)-4(u-\zeta)]|u| d u\right)^{\frac{1}{q}} \\
& =\frac{2^{7}}{3} \Lambda^{-\frac{1}{q}}(\theta, \zeta) D_{1, \Lambda}^{1-\frac{1}{q}}(\zeta, \theta)\left[\left|\psi^{(i v)}(\theta)\right|^{q} D_{2, \Lambda}(\zeta, \theta)+\left|\psi^{(i v)}(\zeta)\right|^{q} D_{3, \Lambda}(\zeta, \theta)\right]^{\frac{1}{q}} .
\end{aligned}
$$


Corollary 2.3. Under the conditions of Theorem 2.3, If we choose $\Lambda(\theta, \zeta)=\theta-\zeta$ then when $\left|\psi^{(i v)}\right|^{q}$ is convex on $K$ for $q \geq 1$ we get

$$
\begin{aligned}
& \left|\frac{(\theta-\zeta)^{2}}{6}\left(\frac{3 \zeta+\theta}{4}\right) f^{\prime \prime \prime}(\theta)-\frac{(\theta-\zeta)}{2}\left(\frac{2 \zeta+\theta}{3}\right) f^{\prime \prime}(\theta)+\frac{\zeta+\theta}{2} f^{\prime}(\theta)-\frac{f(\theta) \theta-f(\zeta) \zeta}{\theta-\zeta}+\frac{1}{\theta-\zeta} \int_{\zeta}^{\theta} f(u) d u\right| \\
\leq & \frac{2^{7}}{3}(\theta-\zeta)^{-1-\frac{1}{q}}(\theta, \zeta) D_{1, \Lambda}^{1-\frac{1}{q}}(\zeta, \theta)\left[\left|\psi^{(i v)}(\theta)\right|^{q} D_{2, \Lambda}(\zeta, \theta)+\left|\psi^{(i v)}(\zeta)\right|^{q} D_{3, \Lambda}(\zeta, \theta)\right]^{\frac{1}{q}},
\end{aligned}
$$

where

$$
\begin{gathered}
D_{1}(\zeta, \theta)=\left\{\begin{array}{c}
\frac{(\theta-\zeta a)^{4}}{4^{4}} \frac{\theta+4 \zeta}{20}, \quad \zeta>0, \frac{3 \zeta+\theta}{4}>0 \\
\frac{(\theta-\zeta)^{4}}{4^{4}} \frac{\theta+4 \zeta}{20}-\frac{\zeta^{5}}{10}, \zeta<0, \frac{3 \zeta+\theta}{4}>0 \\
-\frac{(\theta-\zeta)^{4}}{4^{4}} \frac{\theta+4 \zeta}{20}, \quad \zeta<0, \frac{3 \zeta+\theta}{4}<0
\end{array}\right. \\
D_{2}(\zeta, \theta)=\left\{\begin{array}{c}
\frac{(\theta-\zeta)^{5}}{4^{5}} \frac{5 \theta+19 \zeta}{120}, \quad \zeta>0, \frac{3 \zeta+\theta}{4}>0 \\
\frac{(\theta-\zeta)^{5}}{4^{4}} \frac{5 \theta+19 \zeta}{120}+\frac{\zeta^{6}}{15}, \zeta<0, \frac{3 \zeta+\theta}{4}>0 \\
-\frac{(\theta-\zeta)^{5}}{4^{4}} \frac{5 \theta+19 \zeta}{120}, \quad \zeta<0, \frac{3 \zeta+\theta}{4}<0 \\
\frac{(\theta-\zeta)^{5}}{4^{5}} \frac{\theta+5 \zeta}{120}, \quad \zeta>0, \frac{3 \zeta+\theta}{4}>0 \\
\frac{(\theta-\zeta)^{5}}{4^{5}} \frac{\theta+5 \zeta}{120}-(\theta-\zeta) \frac{\zeta^{5}}{10}-4 \frac{\zeta^{6}}{15}, \zeta<0, \frac{3 \zeta+\theta}{4}>0 \\
-\frac{(\theta-\zeta)^{5}}{4^{5}} \frac{\theta+5 \zeta}{120}, \\
D_{3}(\zeta, \theta)=0, \frac{3 \zeta+\theta}{4}<0 .
\end{array}\right.
\end{gathered}
$$

Remark 2.3. If the mapping $\Lambda$ satisfies condition $C$ then using (2.2) in the proof of Theorem 2.3, then (2.6) becomes the following inequality:

$$
\left|I_{\psi}(\zeta, \theta, \Lambda)\right| \leq \frac{2^{7}}{3} \Lambda^{-\frac{1}{q}}(\theta, \zeta) D_{1, \Lambda}^{1-\frac{1}{q}}(\zeta, \theta)\left[\left|\psi^{(i v)}(\zeta+\Lambda(\theta, \zeta))\right|^{q} D_{2, \Lambda}(\zeta, \theta)+\left|\psi^{(i v)}(a)\right|^{q} D_{3, \Lambda}(\zeta, \theta)\right]^{\frac{1}{q}} .
$$

We note that by use of the preinvexity of $\left|\psi^{(i v)}\right|^{q}$ we get $\left|\psi^{(i v)}(\zeta+\Lambda(\theta, \zeta))\right|^{q} \leq\left|\psi^{(i v)}(\theta)\right|^{q}$. Therefore, (2.7) is better than (2.6).

Corollary 2.4. Taking $q=1$ in Theorem 2.3, then we get

$$
\left|I_{\psi}(\zeta, \theta, \Lambda)\right| \leq \frac{2^{7}}{3 \Lambda(\theta, \zeta)}\left[\left|\psi^{(i v)}(\theta)\right| D_{2, \eta}(\zeta, \theta)+\left|\psi^{(i v)}(\zeta)\right| D_{3, \eta}(\zeta, \theta)\right]
$$

\section{References}

[1] Barani, A., Ghazanfari AG, Dragomir SS. Hermite-Hadamard inequality for functions whose derivatives absolute values are preinvex. Journal of Inequalities and Applications. 2012 (2012):247.

[2] Dragomir, SS. and Pearce, CEM., Selected Topics on Hermite-Hadamard Inequalities and Applications. RGMIA Monographs, Victoria University, 2000.

[3] Hadamard, J., Étude sur les propriétés des fonctions entières et en particulier d'une fonction considerée par Riemann. J. Math. Pures Appl. 58 (1893), 171-215.

[4] Israel, AB. and Mond, B., What is invexity? J. Aust. Math. Soc.Ser. B. 28 (1986), 1-9.

[5] İşcan, İ., Set, E. and Özdemir, ME., On new general integral inequalities for s-convex functions. Applied Mathematics and Computation. 246 (2014), 306-315.

[6] İşcan İ., Ostrowski type inequalities for functions whose derivatives are preinvex. Bulletin of the Iranian Mathematical Society. 40 (2014), 2, 373-386.

[7] Latif, MA. and Dragomir, SS., Some Hermite-Hadamard type inequalities for functions whose partial derivatives in absloute value are preinvex on the co-oordinates. Facta Universitatis (NIŠ) Ser. Math. Inform. 28 (2013), No. 3, 257-270. 
[8] Maden, S., Kadakal, H., Kadakal, M. and İşcan İ., Some new integral inequalities for $n$-times differentiable convex functions. J. Nonlinear Sci. Appl. 10 (2017), 12, 6141-6148.

[9] Matloka, M., On some new inequalities for differentiable $\left(h_{1} ; h_{2}\right)$-preinvex functions on the co-ordinates. Mathematics and Statistics. 2 (2014), 1, 6-14.

[10] Mohan, SR., Neogy, SK., On invex sets and preinvex functions. J. Math. Anal. Appl. 189 (1995), 901-908.

[11] Noor, MA., Hermite-Hadamard integral inequalities for log-preinvex functions. J. Math. Anal. Approx. Theory. 2 (2007), 126-131.

[12] Noor, MA., Invex equilibrium problems. J. Math. Anal. Appl. 302 (2005), 463-475.

[13] Noor, MA., Variational-like inequalities. Optimization. 30 (1994), 323-330.

[14] Pečarić, JE., Porschan, F. and Tong, YL., Convex Functions, Partial Orderings and Statistical Applications. Academic Press Inc., 1992.

[15] Pini, R., Invexity and generalized convexity. Optimization. 22 (1991), 513-525.

[16] Weir, T. and Mond, B., Preinvex functions in multiple objective optimization. J. Math. Anal. Appl. 136 (1998), 29-38.

[17] Yang, XM. and Li, D., On properties of preinvex functions. J. Math. Anal. Appl. 256 (2001), 229-241.

\section{Affiliations}

HURIYE KADAKAL

ADDRESS: Ministry of Education, Bulancak Bahçelievler Anatolian High School, Giresun-Turkey

E-MAIL: huriyekadakal@hotmail.com

ORCID ID: 0000-0002-0304-7192

İMDAT İşCAN

AdDRESS: Giresun University, Dept. of Mathematics, 28100, Giresun-Turkey.

E-MAIL: imdati@yahoo.com

ORCID ID: 0000-0001-6749-0591 